Ciudad y territorio 


\title{
Museo de los desplazados Archivo de procesos de gentrificación
}

\author{
Colectivo Left Hand Rotation
}

Invitados internacionales

Profesionales independientes

hand@lefthandrotation.com

http://www.lefthandrotation.com/

lefthandrotation.blogspot.com

Recibido: julio del 2015

Aceptado: mayo del 2016 


\title{
Resumen
}

El Museo de los Desplazados es una plataforma de colaboración creada por el Colectivo Left Hand Rotation que surge como conclusión del proyecto - taller "Gentrificación No es un Nombre de Señora" en el que se analiza el papel de la cultura en los procesos de gentrificación. El Museo de los Desplazados es un archivo de material de registro de todo aquello que se pierde en los procesos de gentrificación (vídeo, foto, sonido, esquemas de información) y de acciones que impliquen documentación de los procesos de gentrificación y/o acercamiento de la información e interrelación con los perfiles potencialmente en peligro de ser desplazados por procesos de gentrificación.

Palabras clave: desplazamiento; gentrificación; regeneración urbana.

\begin{abstract}
Museo de los Desplazados is a platform created by the Collective Left Hand Rotation arising as completion of the project - workshop "Gentrificación No es un Nombre de Señora" which discusses the role of culture in the processes of gentrification. Museo de los Desplazados is an attempt to record all that is lost to gentrification (video, picture, sound, layout information) and actions that involve documentation of gentrification and interaction with profiles potentially in danger of being displaced by gentrification.
\end{abstract}

Keywords: displacement; gentrification; urban regeneration.

\footnotetext{
Left Hand Rotation es un colectivo artístico en activo desde 2005 que desarrolla proyectos que articulan intervención, apropiacionismo, registro y manipulación de vídeo. El colectivo se estructura como entidad impersonal no asociada al individuo/autor, y aborda cada proyecto bajo la consideración de que la comunidad de recepción no es un espectador, si no parte activa imprescindible en la transformación de la realidad social. La voluntad de las comunidades de testimoniar su situación posibilita la articulación de la acción. En cada una de las acciones del colectivo hay una fuerte consciencia de la importancia del registro audiovisual, tanto por su valor como captura en bruto, como por el potencial de cada clip de vídeo de convertirse en unidades de lenguaje cuya combinación y manipulación posibilita la transmisión de mensajes complejos a partir de detalles del cotidiano. La cámara no puede sino registrar el contexto específico en el que se sitúa. Es a través de esas capturas de lo local que el colectivo reflexiona sobre un sistema global complejo.
} 


\section{Museo de los desplazados Archivo de procesos de gentrificación}

Colectivo Left Hand Rotation ${ }^{1}$

\section{Morir de éxito}

Gentrificación es un proceso de transformación urbana en el que la población original de un sector o barrio deteriorado y con pauperismo es progresivamente desplazada por otra de un mayor nivel adquisitivo, como consecuencia de programas de recalificación de espacios urbanos estratégicos.

Según la historiadora brasileña Otília Arantes, en el proceso de la Revolución Industrial, la cultura dejó de ser un derecho conquistado por los trabajadores para transformarse en el muelle propulsor de la máquina que rige el capitalismo².

La instrumentalización que de la cultura se hace a favor de estos procesos de alteración de la composición social, desarticula la mayoría de las propuestas críticas que se formulan desde la acción artística, fagocitándolas con intereses inmobiliarios, empresariales y financieros. La gentrificación afecta especialmente a la memoria colectiva barrial, imposibilitando la re-construcción del pasado, provocando la pérdida de la identidad local. Los desplazados son los afectados últimos por el proceso de gentrificación.

\section{Glocalización}

Left Hand Rotation entiende que la gentrificación es una respuesta aspiracional de las administraciones públicas locales a una política del desarrollo urbano global cuya finalidad es la implantación de lo denominado como "ciudades marca". No hay dos procesos de gentrificación iguales en su desarrollo por lo que se propone trabajar desde lo local, desde la especificidad que estos procesos alcanzan en cada contexto, zona o ciudad, con el objetivo de identificar las herramientas que cada comunidad genera para abordar el conflicto global. 


\section{Archivo}

Left Hand Rotation identifica la forma de archivo, previa renuncia a su aspiración de definición totalitaria de los elementos y tipologías desplazadas, como herramienta de acercamiento y empatización hacia todo aquello que se pierde en procesos que, como el de la gentrificación, suponen la creación de espacios excluyentes y de segregación social. Frente a la creación de comunidades cerradas, se propone el Museo de los Desplazados como una plataforma abierta, incompleta, en continuo proceso de desarrollo y necesariamente colectiva. Es en esa colectividad también el lugar dónde decidir si es este un archivo de lo que hay que olvidar o recuperar.

\section{Work in progress / trabajo colectivo}

Left Hand Rotation crea el Museo de los Desplazados con la participación de los colaboradores locales que, con sus registros, dan forma a este contenedor, y el valor único de éste son las experiencias personales e irregistrables que cada uno adquiera en el proceso. Cada colaborador desarrolla para cada caso, ciudad o área, sus líneas propias y específicas y conserva la autonomía de llegar a sus propias conclusiones.

Left Hand Rotation trabaja, desde la interrelación con los perfiles potencialmente en peligro de ser desplazados por los procesos de gentrificación, acciones que impliquen documentación de los procesos o acercamiento de la información a esos perfiles.

A continuación se reseñan tres proyectos de registro de documentación de procesos de gentrificación. 


\section{Bilbao, Barrio de San Francisco Colaborador: Left Hand Rotation}

Taller impartido en diciembre de 2010 durante las jornadas AKME (Jornadas de Arte, Cultura y Nuevos Medios) en la Facultad de Bellas Artes de Leioa (UPV/EHU, Bilbao).

Ejemplo paradigmático de la aplicación de los principios de la economía creativa y la ciudad marca, Bilbao inicia a finales de los noventa un proceso de regeneración urbana en el que la cultura juega un importante papel dentro la estrategia de capitalización simbólica de la ciudad, con el Museo Guggenheim como punta de lanza.

El efecto Guggenheim ${ }^{3}$ llega a una de las zonas más deterioradas del centro de la ciudad, el barrio de Bilbao La Vieja o San Francisco. El proceso de gentrificación es claro: originalmente el barrio de rentas bajas e inmigración y con un claro abandono por parte del poder público, es sujeto de las nuevas políticas de revalorización de la ciudad que convierten la zona en un caramelo para el mercado inmobiliario.

Las clases económicamente más vulnerables ven peligrar su permanencia ante la inminente subida de precios de los alquileres como consecuencia del aumento de la demanda de nuevos vecinos (con un importante incremento de la clase creativa) por residir en el barrio, ahora estetizado bajo etiquetas como bohemio y multicultural.

El barrio San Francisco/ Bilbao La Vieja fue elegido como contexto de intervención crítica en el taller Gentrificación no es un nombre de señora.

\section{Acción inaugural:}

Como consecuencia de este primer taller surge la plataforma de colaboración Museo de los Desplazados, cuya "acción inaugural" se realiza en colaboración con los participantes y sirve de homenaje a los desplazados del barrio de Bilbao La Vieja: ficticios antiguos vecinos y habitantes de la zona, que un día se vieron obligados a abandonar el centro de la ciudad, empujados por el proceso de gentrificación.

Se utilizaron para ello réplicas de las placas oficiales de la ciudad que señalan lugares/ escenarios de la presencia o nacimiento de personajes históricos en el lugar. 
PLACA 1: En esta misma acera transcurrían las horas de Joseba Lasuen, indigente y hombre sin fortuna, antes de que el barrio sufriera un proceso de gentrificación por el cual se vio desplazado a otro lugar.

PLACA 2: En este edificio pasó los primeros años de su vida Maite Arrieta, madre y prostituta, antes de que el barrio sufriera un proceso de gentrificación por el cual se vio desplazada a otro lugar.

PLACA 3: En esta casa vivió el ciudadano desconocido Iñaki Zarrabeitia, vecino y jubilado, antes de que el barrio sufriera un proceso de gentrificación por el cual se vio desplazado a otro lugar.

PLACA 4: Estas calles fueron frecuentadas por Begoña Arteche, ama de casa y vecina común de la zona, antes de que el barrio sufriera un proceso de gentrificación por el cual se vio desplazada a otro lugar.

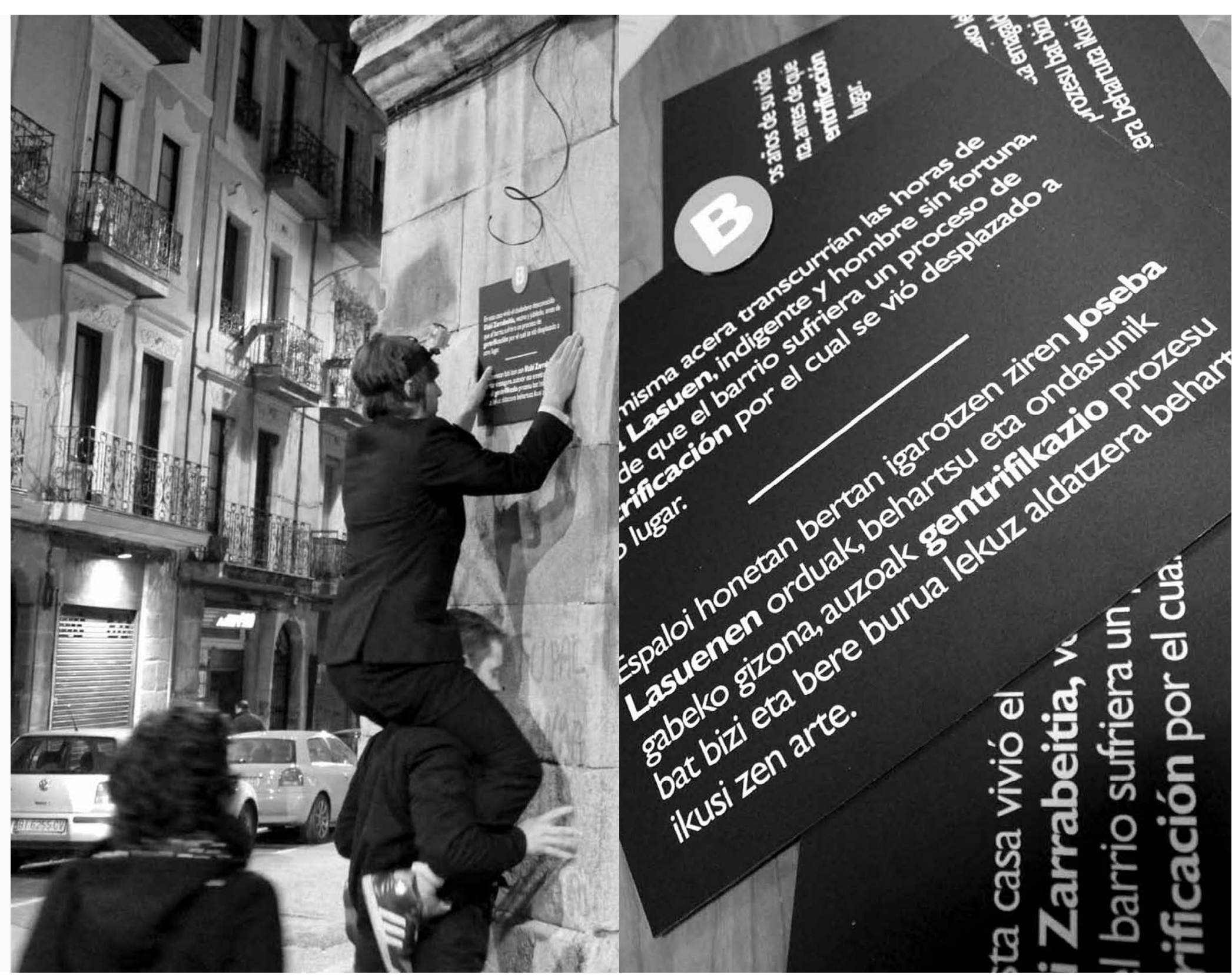

Figura 1. Imagen de la acción realizada para rendir homenaje a los desplazados del barrio de Bilbao La Vieja, misma que consistió en la instalación de réplicas de las placas oficiales con información de personajes históricos en el lugar. 


\section{Madrid, Barrio de Lavapiés}

Taller impartido en Octubre de 2011 en el Espacio Offlimits de Madrid.

Se analizaron los múltiples casos existentes en barrios de Madrid: Tetuán, Arganzuela, Malasaña, Lavapiés; la mayoría aparecidos a raíz del nuevo Plan General de Ordenación Urbana (PGOU).

Madrid aspira desde hace años a ser una nueva ciudad marca, y la política neoliberal de sus gobernantes marca los planes urbanísticos, especialmente en lo que respecta a la rehabilitación de su centro histórico.

Como ya sucediera en otros barrios, Lavapiés sufre un progresivo proceso de gentrificación que tiene en la cultura y la multiculturalidad a sus mejores aliados. El barrio, históricamente inmigrante y popular, ve hoy aumentar el valor de sus rentas, estratégicamente sitiado por grandes espacios culturales: Museo Nacional Reina Sofía, La Casa Encendida, el Centro Dramático Nacional. Lavapiés es también territorio de resistencia, de autogestión, de individuos activos y concienciados. Paradójicamente, este pulso vivo del barrio, a la vez que contrarresta los esfuerzos por incluir Lavapiés en un nuevo espacio conquistado para el turista, no puede evitar ser el atractivo objetivo de un cada vez más popular patrón de consumo que busca en el activismo del barrio la llave de la autenticidad aspiracional.

\section{Kit de negociación de la identidad}

A lo largo de su historia, el barrio de Lavapiés ha ido evolucionando su identidad4: judío y árabe en el S. XV, sede de la "manolería" (mezcla de oriundos e inmigración nacional) y del Madrid castizo hasta el S. XIX, cuna de las primeras casas okupas en los años 80, destino de la diáspora internacional en los años 90. En 1998 Lavapiés es declarado Área de Rehabilitación Integrada, atrayendo estratégicamente a nuevos vecinos al barrio, de poder adquisitivo mayor e interesados en el ambiente bohemio.

Los nuevos y viejos habitantes de Lavapiés tendrán que ser capaces de negociar nuevamente la identidad del barrio, antes de que los intereses económicos y políticos lo hagan por ellos.

Como juego simbólico que representa lo anteriormente descrito, en la jornada práctica 
del taller se distribuyó entre los participantes un Kit de Negociación de la Identidad que incluía:

- Un poster con información de la historia del barrio y material teórico sobre las estrategias de gentrificación.

- Un test de negociación de la Identidad, donde se muestran diferentes discursos identitarios del barrio asociados a grupos que lo habitan (inmigrantes internacionales, activistas, vecinos de toda la vida, nuevos vecinos).

- Un "mapa-relato" del barrio por completar.

Se pidió a los asistentes al taller que completaran un Kit de Negociación de la Identidad junto con una persona del barrio, pasando por las siguientes fases:

Fase A. Ayudar a cumplimentar el "mapa-relato", pidiéndole a la persona que identificase y comentase una serie de puntos subjetivos en el mapa como su recorrido más transitado y las sensaciones mientras lo transitaba o el lugar que más y que menos le gustase del barrio.

Fase B. Cumplimentar el Test de Negociación de la Identidad, entendido como un juego para hablar de las distintas versiones del barrio, dándole a la persona la posibilidad de identificarse o no con esas líneas discursivas.

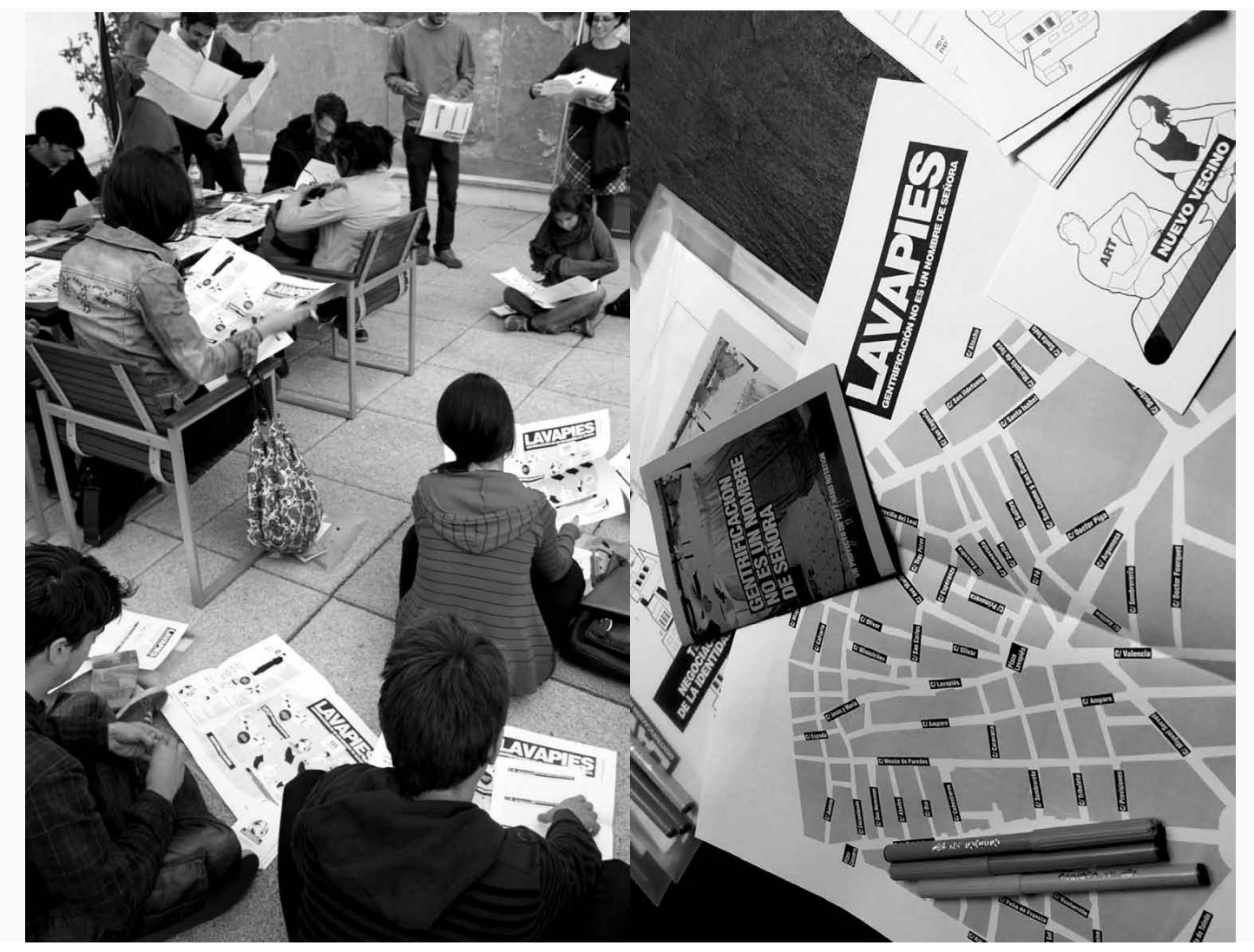

Figura 2. Imagen de la jornada práctica del taller en el que se realiza un juego simbólico para el que se distribuyó entre los participantes un Kit de Negociación de la Identidad que incluía un poster con información de la historia del barrio y material teórico sobre las estrategias de gentrificación así como un test de negociación de la Identidad, donde se muestran diferentes discursos identitarios del barrio asociados a los diversos grupos que habitan el Barrio Lavapiés. 


\section{Valencia, Barrio del Cabanyal}

Taller impartido en en Marzo de 2012 en el Espacio La Clínica Mundana de Valencia.

El Cabanyal, si bien no es un caso de gentrificación propiamente dicho, evidencia una curiosa paradoja: sus vecinos demandan precisamente la llegada de nuevos habitantes para revitalizar una zona con un severo estado de degradación.

\section{Bien de interés cultural vs. bien de interés común}

El Cabanyal ha sufrido durante las últimas décadas un abandono absoluto por parte del poder público, provocando una profunda degradación en sus calles. Esto no hace más que facilitar la implantación del plan urbanístico PEPRI del CABANYAL 2001 (Plan Especial de Protección y Reforma Interior del Cabanyal). A continuación se reseñan tres proyectos de registro de documetanción de procesos de gentrificación. que propone continuar hasta el mar la Avenida Blasco Ibáñez, precisando para ello de la demolición de un $30 \%$ del barrio, cuyos restos quedarían divididos en dos mitades, provocando la destrucción de un área declarada BIC (Bien de Interés Cultural) y la desaparición del patrimonio, memoria e historia de una comunidad, en nombre del bien común.

Irónicamente, la Avenida Blasco Ibáñez lleva el nombre de un autor cuyo legado escrito es en gran parte inspirado por el costumbrismo que el Cabanyal representa a nivel de patrimonio vivo. Es por tanto en su nombre que se borrará la memoria de todo aquello que conformó una gran parte de su obra.

\section{Proyecto: Expolio}

\section{Acción: Parte 1. Testimonios}

Partimos de la obra de Blasco Ibáñez, cuyos libros se inspiran en las costumbres de barrios como El Cabanyal. En esta primera parte realizamos un expolio de decenas de sus obras, partiéndolas por la mitad. La acción se completa en las calles del Cabanyal, entregando a sus vecinos las mitades de los libros a la vez que se les plantea la pregunta: ¿Qué opina usted de la "OBRA" de Blasco Ibáñez?, dicotomía planteada entre la "obra literaria" y la "obra urbanística”, ambas bajo el nombre del mismo escritor.

\section{Acción: Parte 2. Marcapáginas}

Intervenimos bibliotecas y librerías de Valencia, situando en la mitad de los libros de Blasco Ibáñez un marcapáginas con el texto: "Si está a favor del Plan PEPRI rompa este libro por aquí. La división del Cabanyal por parte de la avenida proyectada en el plan 
urbanístico PEPRI imposibilita la comprensión de su identidad, de igual manera que no puede comprenderse el significado de un libro partido por la mitad."

\section{Acción: Parte 3. Fin de la obra}

La "obra urbanística" propuesta por el Ayuntamiento de Valencia de prolongar la Avenida Blasco Ibáñez hasta el mar supone el fin de la "obra literaria" del propio Blasco Ibáñez. En la acción, marcamos el final de la OBRA en su más amplio sentido de la palabra mediante carteles de gran formato.

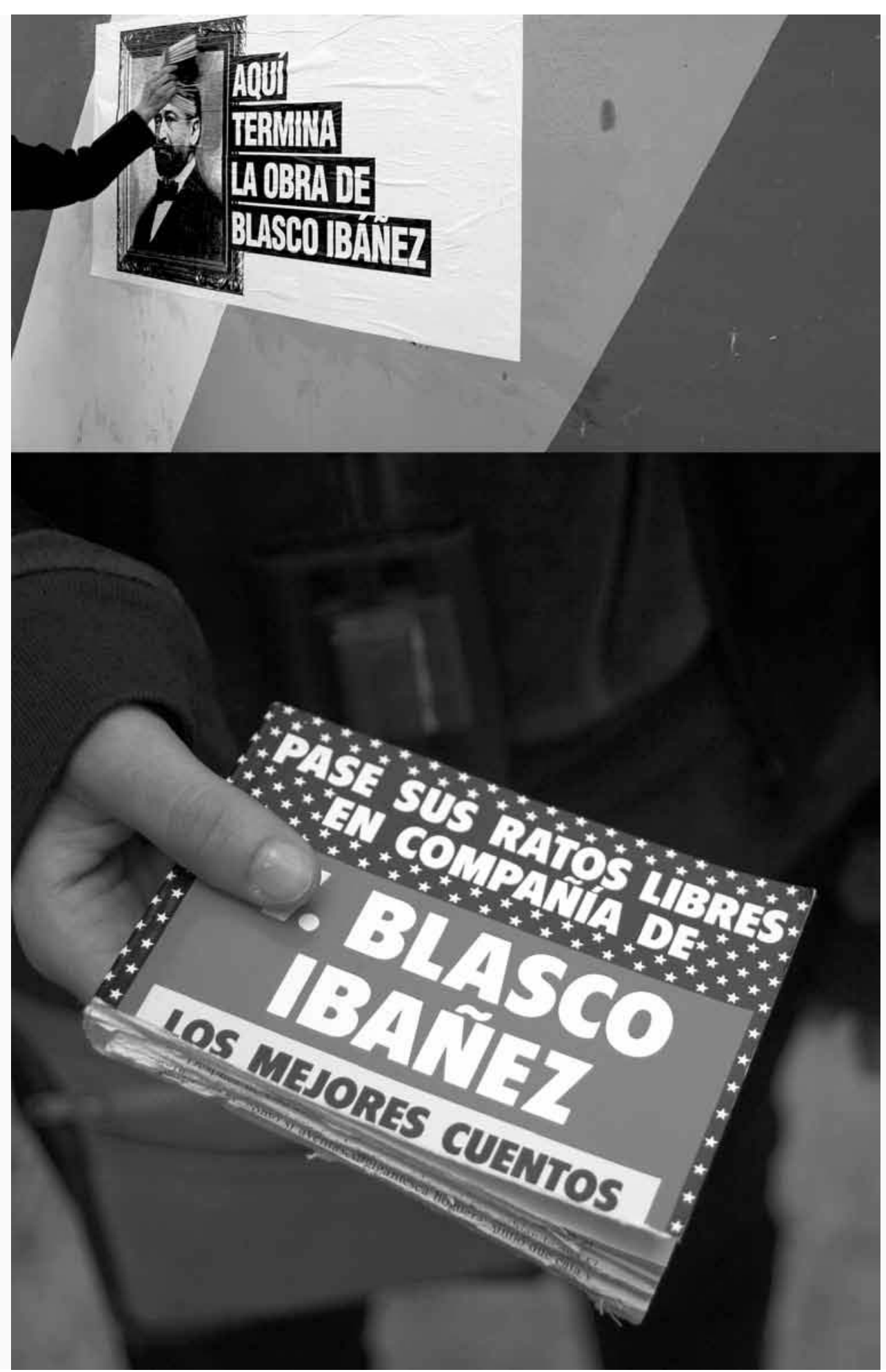

Figura 3. Imagen de la Acción Testimonios que consistió en realizar un expolio de decenas de sus obras, partiéndolas por la mitad, al mismo tiempo que se entregó a los vecinos del Barrio del Cabanyal las mitades de los libros. 


\title{
Referencias
}

Arantes, O; Vainer, C.; Maricato, E. (2000) A Cidade do Pensamento Único. Desmanchando consensos. Rio de Janeiro: Editora Vozes.

Esteban, Iñaki. (2007) El Efecto Guggenheim: del espacio basura al ornamento. Barcelona: Anagrama.

Florida, Richard. (2005) Cities and the Creative Class. New York; London: Routledge.

Lefebvre, Henri. (1968) Le Droit à la ville. Paris: Anthropos.

Muñoz, Francesc. (2010) Urbanalización. Paisajes comunes, lugares globales. Barcelona: Gustavo Gili.

\section{El Colectivo Left Hand Rotation ha participado en:}

\author{
Festivales de videoarte, documental y cine \\ - Pantalla Libre en Matadero (Madrid) 2013 \\ - L’Alternativa, Festival de Cinema Independent de Barcelona 2014 \\ - Festival FIVA (Buenos Aires) 2014 \\ - Jornadas de Reapropiación 2014 (México) \\ - Zinebi Festival internacional de cine documental y cortometraje de Bilbao 2014 \\ - Festival Alcances, Muestra Cinematográfica del Atlántico (Cádiz) 2013 y 2014 \\ - BCCN Barcelona creative commons film festival 2014 \\ - LOOP Festival (Barcelona) 2014 y 2015 \\ - Simultan, festival for video, media art, exploratory music and sound (Rumanía) 2014 \\ - Visiones Contemporáneas, Centro de Arte Contemporáneo DA2 (Salamanca) 2015 \\ - Territorios y Fronteras (UPV Bilbao) 2015 \\ - Ciudades reveladas: Muestra audiovisual de cine y ciudad (Buenos Aires, Argentina) 2015 \\ - Venice International Experimental Cinema and Performance Art Festival 2015 \\ - QCC, Queer Cinema City (Canadá) 2015 \\ - FICSC, Festival Internacional de Cine Social de Concordia (Argentina) 2015 \\ - FETSAC, Festival de la Escuela de Arquitectura de A Coruña 2015
}

\section{Exposiciones}

- Inéditos 2014 en La Casa Encendida (Madrid)

- Estació Creactiva (Tarragona) 2014

- Pickpocket Gallery (Lisboa) 2014

- C.I.T.I. (Centro de Investigación Técnicamente Imprevisible) en la Sala de Arte Joven de la Comunidad de Madrid 2015

- Piso Piloto (CCCB, Barcelona/Medellín) 2015

- TRANSlocaciones (Ars Santa Mónica, Barcelona) 2015 
Este artículo forma parte de:

\section{REVISTARQUIS}

Para más información, artículos, e instructivo de publicación, visite:

www.arquis.ucr.ac.cr/revistarquis.html 